in C.F. antibody titre against adenovirus, influenza $A$ and $B$, parainfluenza 1, 2, and 3 , mumps, or herpes simplex virus.

The slow transmission of infection from case to case in this home for infants suggests that R.S. virus infection may be considerably less contagious than measles or chickenpox. The transmission between the rooms may have been caused by some member of the staff. Two of them had a slight coryza and one had coryza and fever during the investigation. However, in none of our staff members studied, including the three mentioned above, could R.S. virus infection be established. According tc Johnson et al. ${ }^{2}$ reinfection with R.S. virus 1 lay occur and is perhaps quite common.

Dr. Ditchburn and colleagues stated that even when special precautions are taken to exclude infection, such as treatment in cubicles, the cross-infection with R.S. virus is not effectively prevented. One reason is that adults with mild respiratory symptoms may be the vehicle by which R.S. virus is transmitted. I agree fully with this statement, but in my opinion children with acute respiratory illnesses and other infections should be treated in wards especially built for the care of patients with such diseases and with a staff well trained in taking care of children with infections. In Sweden every county has a clinic for infectious diseases in connection with a big general hospital. Most of these Swedish departments for infectious diseases are very modern, with several wards with only single-bed rooms. All these rooms are separately ventilated and each has one sluice (air lock) to the corridor and another one to the outside of the building. The patients are taken into the ward room from the outside only. $X$-rays are taken at the bedside and only exceptionally are patients transported through the corridors. This avoids airborne transmission of infectious agents between patients, but does not avoid transmission of infectious agents from the staff to the patients. as seems to have been the case during the outbreak reported by Dr. Ditchburn and others. If, however, the staff members are well trained in prophylactic measures, and if they are not allowed to work when they are infected, the risk for cross-infection is reduced to a minimum. In this connexion it can be mentioned that we have treated several children with proved R.S. virus infection in our paediatric wards for infectious diseases without any spread to other hospitalized children.-I am, etc.,

GÖRAN STERNER

University Clinic for Infectious Diseases, Danderyd Hospital,

1 Sterner, G., Wolontis, S., Bloth, B., and de Hevesv, G., Acta Paediatrica Scandinavica, 1966, 55,273 .

2 Johnson. K. M., Bloom, H. H. Mufson, M. A., Medicine, 1962, 267, 68.

\section{Stroke Units}

SIR,-I was interested in your leading article (6 November, p. 313) suggesting the creation of "regional stroke units." I was interested too in the comments of Dr. Bernard Isaacs (20 November, p. 492). Geriatricians such as Professor George Adams of Belfast and Dr. Bernard Isaacs himself have undertaken pioneer work during the past few years in the field of stroke rehabilitation.
For too long stroke rehabilitation has been one of the Cinderellas of medicine in general and of neurology in particular. Stroke is the commonest cause of severe chronic disability in the community. ${ }^{1}$ A considerable amount of research has been done into problems of pathology, hypertension, changes in cerebral blood flow, and the place of anticoagulants in the prevention of strokes. Despite all the experimental work that has been done a large number of previously active independent people are rendered useless and frequently completely dependent upon others as a result of a stroke. Research into the problem of recovery after stroke has lagged very seriously behind research into other fields. This is perhaps surprising when it is recalled that strokes present a very common problem having enormous financial and personal implications.

There are probably between 100,000 and 120,000 new strokes each year in England and Wales. ${ }^{2}$ Approximately half the people who have a stroke will survive the first month. This means that in England and Wales approximately 60,000 people per year will survive the acute episode. We estimate that between 30,000 and 35,000 of these will have a hemiplegia. It appears that about $25 \%$ of non-fatal strokes are admitted to hospital each year-approximately 15,000 . If these patients are kept in hospital for an average of 6-8 weeks at a weekly cost of $£ 70$ the cost to the nation of this problem alone will be over $£ 6,000,000$. In addition, there is the enormous cost of patients admitted to hospital who are never discharged. In making these calculations it must be said that there is very little published information about the epidemiology and natural history of cerebrovascular disease in the general population of England and Wales, though there are several excellent hospital studies.

A recent personal study in a general hospital in the South West showed that the average acute stroke inpatient received approximately half an hour's physiotherapy a day. The average amount of physiotherapy given to acute stroke patients who attended on an outpatient basis was one hour per week. A similar situation existed with speech therapy. An investigation undertaken in the same general hospital showed that the average acute dysphasic patient received ten minutes speech therapy a day. In another hospital in the same area no speech therapy was available at all. We have thus reached the situation in which patients are admitted ostensibly for rehabilitation and yet are given very little. All too frequently the patient spends his time sitting by his bed staring into space. I suspect that a similar situation exists in many acute district general hospitals throughout the country.

It seems likely that if we are to achieve reasonable results, it will be necessary to provide better and more intensive rehabilitation facilities than at present exist. $\mathrm{My}$ personal experience is that the rehabilitation programmes offered by district general hospitals are unsatisfactory. Most physiotherapy departments have neither the facilities nor the staff with which to provide the necessary treatment programme. Furthermore, some physicians doubt the value of physiotherapy and other rehabilitation procedures, as it must be conceded that very little objective work has been done on the question of the influence of therapy on recovery in the damaged central nervous system.
I submit that there is a strong case for the setting up of one or more experimental stroke units which would attempt to delineate the size of the problem, to ascertain the effect of rehabilitation techniques upon recovery, and to develop new rehabilitation methods.-I am, etc.,

Bristol

R. LANGTON HewER

1 Harris, A. I., Cox, E., and Smith, C.H.W., Handicapped and Impaired in Great Britain,
Office of Population Censuses and Surveys, 2 Modern Geriatrics, 1971, 1, 368.

\section{Tetracycline and Renal Function}

SIR,-May I add to Dr. C. B. Brown's timely warning about the use of tetracycline in the presence of renal failure (13 November, p. 428). In addition to the antianabolic effect of this drug there are other considerations.

The biological half-life of tetracycline is approximately 8.5 hours in patients with normal renal function. This is prolonged to between 57 and 108 hours in patients with severe renal failure. ${ }^{1}$ The administration of tetracycline in doses of $1000 \mathrm{mg}$ a day to patients with renal failure will lead not only to a rise in blood urea due to the antianabolic effect but also to accumulation of the drug. This is often associated with an increased incidence of gastrointestinal side effects including vomiting and/or diarrhoea. The resulting sodium and water depletion may then precipitate a patient with stable chronic renal failure into severe uraemia.

The antianabolic effect of tetracycline seems to be shared by oxytetracycline, lymecycline, and demethylchlortetracycline. ${ }^{2}$ Little and Bailey have shown, however, that the administration of doxycycline to patients with varying degrees of renal failure does not lead to a rise in the blood urea level. ${ }^{2}$

The mean biological half-life of doxycycline in patients with normal renal function has been reported as 18 hours $^{3}$ and 22 hours. ${ }^{4}$ Satisfactory serum levels can be achieved in patients with normal renal function by a loading dose of $200 \mathrm{mg}$ followed by $100 \mathrm{mg}$ once daily thereafter. Mahon, Wittenberg, and Tuffnel $^{3}$ have shown that no accumulation of doxycycline occurs in patients with severe renal failure given the same dosage schedule for up to 15 days. My own unpublished observations confirm the results of Little and Bailey that the administration of doxycycline to patients with renal failure does not lead to a rise in the blood urea level. In addition, the biological half-life of doxycycline in one anephric patient and three patients with endogenous creatinine clearances of $<5 \mathrm{ml} /$ min was found to be similar to the reported estimates for patients with normal renal function (13-19 hours)

Further investigation of the effects of doxycycline administration on renal function, nitrogen balance, and sodium balance in patients with renal failure are clearly indicated, but the evidence already available suggests that doxycycline may prove to be of particular value when a broad spectrum antibiotic is required in patients with renal failure.-I am, etc.

Department of Medicine,

J. R. CURTIS

Fulham Hospital,

1 Kunin, C. M., Proceedings of the International Congress on Nephrology, Washington, 1966. 
2 Little, P. J., and Bailey, R. R., New Zealand Medical fournal, 1970, 72, 183.
Mahon, W. A., Wittenberg, J. V. P., and Tuffnel, P. G., Canadian Medical Association fournal,

Fabre, J., Pitton, J. S., and Kunz, J. P., Chemo-

\section{Hypoxic Newborn Infants}

SIR,-The paper by Dr. M. A. Chadd and others (27 November, p. 516) appears to lend support to other work on the effects of birth asphyxia on haemostasis in the new born. ${ }^{1}$ However, in fairness to themselves I feel that the authors should correct a number of inconsistencies which occur in their paper as published.

The study purports to be on full-term infants, but yet in Table $I$ the mean gestation of the hypoxic group is given as 35.1 weeks and that of the controls as 35.5 weeks. Antenatal complications (Table I) are recorded as $46 \pm 10 \cdot 2 \%$ (hypoxic) and $17 \pm 7.8 \%$ (control). Surely antenatal complications were or were not present in each case? There is no mention of what antenatal complications were recorded.

Rectal temperature below $35^{\circ} \mathrm{C}$ in the firs 24 hours (Table $I$ ) is recorded as $4 \pm 4 \cdot 1 \%$ for the hypoxic group and $25 \pm 8.8 \%$ for the control group. Again it is difficult to see what these figures mean and any interpretation put on them seems incompatible with the text statement that a rectal temperature below $35^{\circ} \mathrm{C}$ was detected in a greater proportion of hypoxic babies (as compared with control babies).

The statement in the discussion that this work suggests that "disseminated intravascular coagulation is a frequent factor of major importance in infants who die shortly after birth with haemorrhage" hardly seems justified in a paper devoted to babies with low Apgar scores in whom "there was no overt evidence of haemorrhage." Finally, the statement in the summary that "the consumption of clotting factors ... led to the appearance of an haemorrhage diathesis" is at variance with the clinical findings reported.-I am, etc., Institute of Child Health,

J. S. Wigglesworth London W.12

1 Chessells, J. M., and Wigglesworth, J. S.,

\section{Plastic Stitch Holder}

SIR.-During the operation of anterior re section of the rectum interrupted stitches are usually used for anastomosing the bowel. The ends of each stitch are left long and held with artery forceps, to be tied at the end when all the stitches are in position. The assistant has to hold the forceps in order and on a slight stretch so that the thread does not get tangled or twisted.

A plastic stitch holder may be used for this purpose. This will leave the assistant's hand free. The stitch holder is pinned to the green sheet covering the patient, using a safety-pin and placed in a suitable position near the edge of the wound. The ends of each stitch are slipped in a slit in the stitch holder (Fig.)

The plastic stitch holder can be made from the disposable plastic container of a Foley's catheter; approximately $18-20 \mathrm{~cm}$ length from the sealed end of the plastic container is ideal for this purpose. The sealed

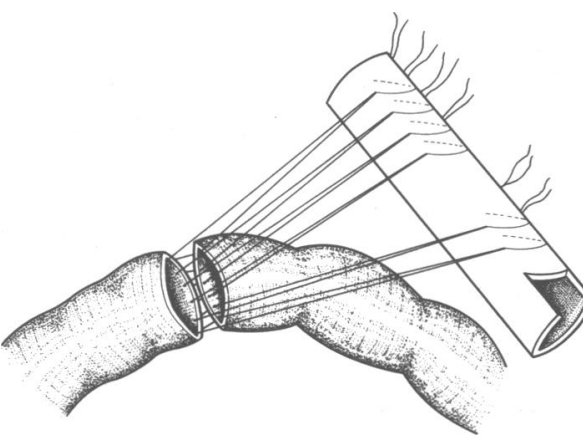

I would like to record for the information of your readers that unless satisfactory answers are given by the authors to the following questions the diagnosis of "intestinal infarction in a straightforward sickle-cell trait" is at best very shaky indeed.

(1) Apart from "acute abdominal pain" and "board-like rigidity of the whole abdomen" what were the other clinical features? Was there history of cold-season rheumatism? Was the patient jaundiced or clinically anaemic?

(2) How much of $A_{2}, A$ and $S$ haemoglobins were there? How did they exclude sickle-cell beta-thalassaemia?

(3) Was there a tendency to peculiar end is more suitable than the open end, because it is less malleable. Slits are cut in one of the rounded surfaces of the tube with a scalpel blade. Each slit should be slightly curved and slanting, or oblique so that the thread will not slip out from it easily.

I should like to thank Mr. D. V. Kneafsey for trying the device during operations and Dr. M. Bodkin for the original drawing. -I am, etc.,

Merlin Park Regional Hospital,

A. MikhaII

Galway

\section{Size of Record Folder}

SIR,-Dr. J. K. Hawkey and others (11 December, p. 667) favour the A4 folder, and their project, financed by the Department of Health and Social Security, was enhanced by a motion supporting the A4 size for general practice at the annual general meeting of the Royal College of General Practitioners on 20 November, as a reference to council.

The present M.R.E. (5 or 6) takes all sheets including quarto, or foolscap, or A4, folded once or twice only. The trouble in using it is not its size but the organization and methods of filing.

Some hospitals still fold letters to fit a window in the envelope to save typing time. This causes much unfolding and refolding when received, or none at all and stuffing in the M.R.E. as it is. There is no positive policy, such as described in the college's journal, 1 adopted by the Department of Health at executive council level, and such negative policies as there are (selective destruction, etc.) have never been published to show why the present M.R.E. was adopted in 1948, from its German original of 1910.

It needs expert attention to detail but is the right size and shape for practical politics for 50 million people.-I am, etc.,

\section{St. Albans, Herts}

M. J. JAMESON 1 Jameson, M. J., foumal of the College of General
Practitioners, 1966, 11, 336.

\section{Sickle-cell Trait and Altitude}

SIR,-The diagnosis of intestinal infarction during flight in a Ghanian nurse with positive sickling is so important that it is surprising to find that all Dr. R. L. Green and colleagues (4 December, p. 593) could offer by way of laboratory confirmatory tests was the statement: "Haemoglobin studies later showed that she was a sickle-cell trait carrier." rouleaux formation of the red cells? What, in fact, were the haemoglobin level and platelet count? Was she polycythaemic?

(4) What about her G-6-PD? Was she deficient in this enzyme or normal?

While I wondered seriously how the three authors, who to my knowledge have never worked in Ghana, obtained such a poorly from the acknowledgements the name of a British surgeon who worked in the same hospital as myself. Could the authors please give further details about the patient described in Case I? Perhaps we could trace the patient and undertake a fuller investigation including a family study. This would help clear up a matter which has serious international repercussions.-I am, etc.

Military Hospital,

R. O. ADDAE Accra

\section{Gastritis and L-Dopa}

SIR,-Though the use of L-dopa in Parkinson's disease is now established in Britain its effectiveness is frequently limited by side effects, of which gastrointestinal disturbances are the most common. While gastrointestinal bleeding is not mentioned in most recorded series of patients on levo-dopa, Keenan's report of five examples of this complication in the Eaton collaborative study of 485 cases suggests that it may be a rare sequel to L-dopa therapy. I have recently observed gastric bleeding in a patient taking this drug

A man of 56 , whose past history was unexceptional except for a myocardial infarction in 1958, developed Parkinson's disease in 1964. The disorder gradually progressed and by the time of his admission to hospital in 1970 he was severely incapacitated, and showed the typical face, voice, and posture of advanced Parkinsonism with rigidity and bradykinesia predominating. He was started on a small dose of L-dopa, which was gradually increased to $2 \mathrm{~g}$ a day, on which dose he was discharged together with orphenadrine which he had been taking for several years. Five weeks later he had an acute painless melaena. There was no evidence of either haemorrhage from other sites or a bleeding diathesis. Laparotomy revealed diffusely haemorrhagic and swollen gastric mucosa and a pyloroplasty was performed. The histological picture was that of a nonspecific gastritis. He made an uninterrupted recovery and while continuing on orphenadrine was given amantadine in place of the levo-dopa. Five months later he died of a myocardial infarct. Postmortem examination of the stomach revealed that the previous inflammation had been replaced by diffuse mucosal scarring and submucosal fibrosis. investigated case from Ghana, I recognized 